\title{
INFORMATION FUSION BY EXTENDED POWER-BASED AGGREGATION IN IMAGE SEGMENTATION
}

\author{
Endre Pap ${ }^{1, *}$, \\ Marija Delić2, \\ Ljubo Nedović2
}

${ }^{1}$ Singidunum University,

Belgrade, Serbia,

2University of Novi Sad,

Faculty of Technical Sciences,

Department of Fundamentals Sciences,

Novi Sad, Serbia

\begin{abstract}
:
In this paper we construct two extended aggregation functions, extended powers product and extended weighted arithmetic mean of powers. Their basic properties are examined, as well as certain properties of distance functions constructed by them. In addition, one pixel descriptor, which is motivated by Local Binary Pattern family of descriptors (LBPs), is introduced. We give an application of the introduced extended aggregation functions and descriptor, by a construction of a new distance function, used in Fuzzy c-Means Clustering Algorithm (FCM) for image segmentation.
\end{abstract}

\section{Keywords:}

extended aggregation function, distance function, image segmentation, pixel descriptor, fuzzy c-Means algorithm.

\section{INTRODUCTION}

The procedure of combining several values into a single representative one is called aggregation, and the function performing this process is called aggregation function, see [1], [2], [3], [4]. In this paper we introduce two extended aggregation functions which change their values of weights depending on their number of variables and we apply them on distance functions. With appropriate selection of the aggregation function and the initial distance functions, it is possible to model some of the desired properties of the newly constructed distance function, which we apply in an image segmentation algorithm. Distance functions have basic role in image processing, see [5], [6], [7], [8], [9], [10], [11], [12], [13]. In image segmentation algorithms, distance functions represent a decision criterion for grouping pixels into segments, see [6], [8], [14], [15], [11]. In this paper we investigate the construction of distance functions and metrics using special aggregation functions, which we further apply in image segmentation. This paper is a continuation of the research carried out in [15], [16], [11]. With an appropriate choice of aggregation function, we model the criterion used to distinguish two objects by constructed distance function, see [11]. There are several clustering algorithms suitable for tasks considered in this paper, and among all we select FCM for this purpose. Although it does not belong to the class of fast algorithms, it gives good results regarding the compactness of the obtained
Correspondence:

Endre Pap

e-mail:

epap@singidunum.ac.rs 
segments, and it is widely accepted in practice. In Section II, definitions and properties of distance functions and aggregation functions relevant for our research are listed. In Section III extended aggregation function is defined. Then in Section IV two new extended aggregation functions, named extended powers product and extended weighted arithmetic mean of powers, are introduced and their properties are examined. In Section $\mathrm{V}$ we introduce one new pixel descriptor, which is used in the segmentation criterion. This descriptor is a modification of LBP descriptor, see [17], [18], motivated by one fuzzyfied LBP member $\alpha$-LBP, see [19]. Section VI is devoted to an application of the introduced extended aggregation functions and descriptor for distance function construction, which is used in image segmentation. In the evaluation of the presented method, one image is used. For more experimental details, images and segmentation results see [20].

\section{PRELIMINARIES}

We give here some basic concepts related to distance functions and aggregation functions.

Definition 1: Let $X$ be an arbitrary non-empty set. The distance function in set (space) $X$ is a function $d: X^{2} \rightarrow[0, \infty[$, which have the following properties:

$$
\text { (d01) } \forall x, y \in X, d(x, y)=d(y, x) \quad \text { (symmetry), }
$$

(d02) $\forall x \in X, d(x, x)=0$

(reflexivity).

Ordered pair $(X, d)$ is a distance space.

Definition 2: On a non-empty space $X$, distance function $d: X^{2} \rightarrow[0, \infty[$ may have following additional properties:

(d03) $\forall x, y \in X, d(x, y)=0 \Rightarrow x=y$,

(d04) $\forall x, y, z \in X, d(x, z) \leq d(x, y)+d(y, z)$

(triangle inequality).

A distance function $d: X^{2} \rightarrow[0, \infty[$ is a metric on the set $X$ if it satisfy the implication (d03) and the triangle inequality (d04).

We start with a basic aggregation function, the arithmetical mean $A M:[0,1]^{n} \rightarrow[0,1]$ of $n$ numbers $x_{1}, x_{2}, \ldots, x_{n} \in[0,1]$, given by

$A M\left(x_{1}, x_{2}, \ldots, x_{n}\right)=\frac{x_{1}+x_{2}+\ldots+x_{n}}{n}=\frac{1}{n} \sum_{i=1}^{n} x_{i}$.
Basic property of the arithmetical mean is the monotonicity, i.e., if in the arithmetical mean $\frac{x_{1}+x_{2}+\ldots+x_{n}}{n}$ we take instead of the number $x_{n}$ a greater number $x_{n}^{\prime}$ , then the new arithmetical mean $\underline{x_{1}+x_{2}+\ldots+x_{n}^{\prime}}$ will be greater than the previous one. The second basic property of the arithmetical is the following. The arithmetical mean of $\mathrm{n}$ zeros is again zero and the arithmetical mean of $\mathrm{n}$ ones is again one. Just these properties characterize the general aggregation functions, see [1].

Definition 3: A function $A:[0,1]^{n} \rightarrow[0,1]$ is called an aggregation function in $[0,1]^{n}$ if

1. $A$ is nondecreasing: for $x_{1} \leq x_{1}^{\prime}, \ldots, x_{n} \leq x_{n}^{\prime}$ we have

$A\left(x_{1}, x_{2}, \ldots, x_{n}\right) \leq A\left(x_{1}^{\prime}, x_{2}^{\prime} \ldots, x_{n}^{\prime}\right) ;$

2. A satisfies the boundary conditions

$$
A(0,0, \ldots, 0)=0 \text { and } A(1,1, \ldots, 1)=1 \text {. }
$$

Example 4: Some basic aggregation functions are listed below (for many of them see [1]):

1. Geometric mean: $\operatorname{GM}\left(x_{1}, \ldots, x_{n}\right)=\left(\prod_{i=1}^{n} x_{i}\right)^{1 / n}$.

2. Harmonic mean: $H M\left(x_{1}, \ldots, x_{n}\right)=\frac{1}{\frac{1}{n} \sum_{i=1}^{n} \frac{1}{x_{i}}}$.

3. Minimum: $\operatorname{Min}\left(x_{1}, \ldots, x_{n}\right)=\min \left(x_{1}, \ldots, x_{n}\right)$.

4. Maximum: $\operatorname{Max}\left(x_{1}, \ldots, x_{n}\right)=\max \left(x_{1}, \ldots, x_{n}\right)$.

There is a general classification of aggregation functions, see [1]:

1. Conjunctive: $0 \leq A \leq \operatorname{Min}$;

2. Internal (means): $\operatorname{Min} \leq A \leq \operatorname{Max}$;

3. Disjunctive: $\operatorname{Max} \leq A \leq 1$;

4. Mixed.

\section{EXTENDED AGGREGATION FUNCTIONS}

The notion of aggregation for various number of input values leads us to the definition of extended aggregation function, see [21], [1].

Definition 5: (Extended aggregation function): An extended aggregation function is a mapping $A: \bigcup_{n=1}^{\infty}[0,1]^{n} \rightarrow[0,1]$ 
such that the restriction $A_{[n]}:[0,1]^{n} \rightarrow[0,1]$ of this mapping, for every $n \in N$, is an $n$-ary aggregation function.

Let $A: \bigcup^{\infty}[0,1]^{n} \rightarrow[0,1]$ be an extended aggregation function. Let $X$ be an arbitrary nonempty set, and $d_{i}: X^{2} \rightarrow[0,1]$, $i \in N$, be a family of bounded function. Let us consider the function $d: X^{2} \times N \rightarrow[0,1]$ defined by

$d(x, y ; n)=A_{[n]}\left(d_{1}(x, y), \ldots, d_{n}(x, y)\right)$,

for all $x, y \in X$ and $n \in N$. For $n \in N$ we have

$d_{[n]}(x, y)=d(x, y ; n), \quad \forall x, y \in X$

is a function that maps $X^{2}$ into [0,1] range, same as all above defined functions $d_{i}$. It is interesting to consider, which properties the new constructed functions $d_{[n]}, n \in N$, inherit from functions $d_{i}, i \in\{1,2, \ldots, n\}$, depending on the applied aggregation function.

\section{EXTENDED WEIGHTED ARITHMETIC MEAN OF POWERS}

First we introduce one new extended aggregation function, which we call extended powers product. This function is a generalization of the extended product aggregation function, see [1]. Its properties are examined in details in [20].

Definition 6: (Extended powers product): For arbitrary family of non-negative coefficients

$$
\omega=\left\{\omega_{n, i}>0 \mid n \in N, i \in\{1, \ldots, n\}\right\},
$$

function $\prod_{\omega}: \bigcup_{n=1}^{\infty}[0,1]^{n} \rightarrow[0,1]$ defined by

$$
\prod_{\omega}\left(a_{1}, \ldots, a_{n}\right)=\prod_{i=1}^{n} a_{i}^{\omega_{n, i}}
$$

for $n \in N$, and $\left(a_{1}, \ldots, a_{n}\right) \in[0,1]^{n}$, is called extended powers product. For the family of non-negative coefficients $\omega$ with the property $\forall n \in N, \sum^{n} \omega_{n, i}=1$, operator $\prod_{\omega}$ is called extended 1-powers product and denoted by $\prod_{|\omega|}$.

\section{Example 7:}

1. For the family of coefficients $\omega$ that satisfy $\omega_{n, i}=1, \forall i \in\{1, \ldots, n\}$, for all $n \in N, \Pi_{\omega}$ is an extended product operator $\Pi$.

2. Exceptionally, for the family of coefficients

$$
\omega_{n, i}=\frac{2 i}{n(n+1)}, n \in N, i \in\{1, \ldots, n\},
$$

extended powers product operator $\Pi_{\omega}$ is also called extended weighted geometric mean, and noted as $G_{\Delta}$. Extended weighted geometric mean $G_{\Delta}$ is a special extended 1-powers product aggregation function.

We introduce another new extended aggregation function, which we call extended weighted arithmetic mean of powers. The experimental results presented in [20], and partly in Section VI, show that this operator is very suitable for application in image segmentation.

Definition 8: (WAMP): For families of coefficients

$$
\begin{aligned}
& \omega=\left\{\omega_{n, i}>0 \mid n \in N, i \in\{1, \ldots, n\}\right\}, \\
& \lambda=\left\{\lambda_{n, i} \geq 0 \mid n \in N, i \in\{1, \ldots, n\}, \sum_{i=1}^{n} \lambda_{n, i}=1\right\},
\end{aligned}
$$

function $W A M P_{\lambda, \omega}: \bigcup_{n=1}^{\infty}[0,1]^{n} \rightarrow[0,1]$ given by

$W A M P_{\lambda, \omega}\left(a_{1}, \ldots, a_{n}\right)=\sum_{i=1}^{n} \lambda_{n, i} a_{i}^{\omega_{n, i}}$,

for $n \in N$, and $\left(a_{1}, \ldots, a_{n}\right) \in[0,1]^{n}$, is called extended weighted arithmetic mean of powers.

The function $W A M P_{\lambda, \omega}$ is an extended aggregation function. Namely, function is monotonically nondecreasing in each component, since it is a composition of monotonically non-decreasing functions.

Example 9: For coefficients

$$
\omega=\left\{\omega_{n, i}=1 \mid n \in N, i \in\{1, \ldots, n\}\right\},
$$

the operator $W A M P_{\lambda, \omega}$ is reduced on extended weighted arithmetic mean $W A M_{\lambda}$ operator, see [20].

\section{DESCRIPTORS}

In this section we introduce one pixel descriptor, which will be used in the construction of distance functions that have application in image segmentation, presented in Section VI. In gray-scale images, pixel is 8-bit, i.e., 1-byte nonnegative number from the $0, \ldots, 255$ range, which represents the gray-scale level. In color images, the color of the pixel is a mixture of certain basic color components. The intensity of each component is presented as 8 -bit non-negative number from the $0, \ldots, 255$ range. The pixel of the color image in RGB technique is written by 3 bytes and it is a mixture of red (R), green $(G)$ and blue (B) component, respectively. Besides the color or gray-scale compo- 
nent, other features relevant from the aspect of automatic image processing can be assigned to a pixel, e.g., saturation of color component, or characteristics that describes relations between adjacent pixels. In the literature such characteristics are known as descriptors, see [22]. For gray-scale image (for color image, for more details, see [20]) we have for $m \times n$ pixels in size, with the matrix of pixels $S=\left\lfloor p_{i, j}\right\rfloor_{m \times n}$ The pixel in $i$ i-th row and $j$-th column is marked as $p_{i, j}=\left(s_{i, j}\right)$, where $s_{i, j} \in\{0, \ldots, 255\}$ is the gray-scale level. For the pixel $p_{i, j}$, its closest by the spatial distance neighboring pixels are observed. Let us mark the central pixel with $p=p_{i, j}$, and its neighbors as follows: $n_{1}=p_{i-1, j-1}$, $n_{2}=p_{i-1, j}, n_{3}=p_{i-1, j+1}, n_{4}=p_{i, j+1}, n_{5}=p_{i+1, j+1}$ , $n_{6}=p_{i+1, j}, n_{7}=p_{i+1, j-1}$ and $n_{8}=p_{i, j-1}$. Arbitrary $p_{i, j}, i \in\{2, \ldots, m-1\}, j \in\{2, \ldots, n-1\}$, in the interior of the image, has 8 neighbors that are shown in Figure 1.

\begin{tabular}{|l|l|l|}
\hline$n_{1}$ & $n_{2}$ & $n_{3}$ \\
\hline$n_{8}$ & $p$ & $n_{4}$ \\
\hline$n_{7}$ & $n_{6}$ & $n_{5}$ \\
\hline
\end{tabular}

Fig. 1. Neighbors for a pixel in the interior of the image

Let $\alpha \in\{0, \ldots, 255\}$ be a predefined threshold value, which represents the limit measure for the similarity of two pixels in their colors or gray-scale levels. Let us first define the indicator of $\alpha$-similarity for the central pixel $p=p_{i, j}$ and each of its neighbors $n_{k}, k \in\{1, \ldots, 8\}$ . Indicator value 1 denotes that the color difference is bigger than the previously selected $\alpha \in\{0, \ldots, 255\}$ , otherwise indicator value is 0 , as a measure of $\alpha$ -similarity for central pixel and its neighbor.

Definition 10: For an arbitrary $p_{i, j}$, the selected threshold value $\alpha \in\{0, \ldots, 255\}$, and each of the neighboring pixels $n_{k}, k \in\{1, \ldots, 8\}, \alpha$-similarity indicator $I_{i, j ; \alpha}(k) \in\{0,1\}$ is defined by

$I_{i, j ; \alpha}(k)=\left\{\begin{array}{ll}0, & \left|p_{i, j}-n_{k}\right| \leq \alpha \\ 1, & \left|p_{i, j}-n_{k}\right|>\alpha\end{array}, \quad k \in\{1, \ldots, 8\}\right.$,

where $p_{i, j}, n_{k} \in\{0, \ldots, 255\}$ are single-byte gray-scale levels of pixels. For the pixel $p_{i, j}$ that is on the edge or in the corner of the image and hence does not have some of the neighbors $n_{k}$, we define $I_{i, j ; \alpha}(k)=1$.

\section{APPLICATION IN IMAGE SEGMENTATION}

We give an application of extended weighted arithmetic mean of powers aggregated distance function in image segmentation through FCM algorithm. By using FCM, one color image is segmented. Next to the images, input parameters of the algorithm are the following.

- Number $c$ of clusters that are going to be acquired by segmentation, we select $c=4$.

- Weight coefficient $m$, which affects the quality and speed of segmentation. This parameter is intuitively selected. From the literature, it is known and experimentally confirmed that values around 2.0 give good performance. Hence, we choose $m=2.0$ in our experiments.

- Distance function $d: P^{2} \rightarrow[0, \infty[$, which defines mathematical model for desired segmentation criterion, where $P$ is the set of pixels. For pixels $p_{1}, p_{2} \in P$, value $d\left(p_{1}, p_{2}\right) \in[0, \infty[$ represents a measure of the difference or dissimilarity between them. In order to compare the achieved results and the used distance functions, all distance functions are normalized to the corresponding mapping in the $[0,1]$ range, i.e., $d\left(p_{1}, p_{2}\right) \in[0,1]$.

Some values that represent a measure of the quality of the obtained segmentation and the speed of the algorithm are listed below. These values are the output parameters generated by the segmentation algorithm.

- PI - performance index, measures the compactness of the clustered data. The lower value of this coefficient means a stronger grouping of pixels, i.e., more compact clusters.

- IT - the number of preformed iterations.

- RT - the execution time of the algorithm, in seconds.

For the families of coefficients, $\omega=\left\{\omega_{i}>0 \mid i \in\{1, \ldots, 5\}\right\}$, $\lambda=\left\{\lambda_{i} \geq 0 \mid i \in\{1, \ldots, 5\}, \sum_{i=1}^{5} \lambda_{i}=1\right\}$, a new distance function $d_{\lambda, \omega}: P^{2} \rightarrow[0,1]$ is constructed by applying WAMP operator on the distance functions $d_{1}$ and $d_{2}$ :

$$
\begin{aligned}
& d_{\lambda, \omega}\left(p_{1}, p_{2}\right)=\lambda_{1} d_{1}^{\omega_{1}}\left(r_{1}, r_{2}\right)+\lambda_{2} d_{1}^{\omega_{2}}\left(g_{1}, g_{2}\right)+ \\
& +\lambda_{3} d_{1}^{\omega_{3}}\left(b_{1}, b_{2}\right)+\lambda_{4} d_{2}^{\omega_{4}}\left(s_{1}, s_{2}\right)+\lambda_{5} d_{2}^{\omega_{5}}\left(q_{1}, q_{2}\right)
\end{aligned}
$$

for pixels $p_{i}=\left(r_{i}, g_{i}, b_{i}, s_{i}, q_{i}\right) \in P, i \in\{1,2\}$ (for more details see [20]), where normalized distance function $d_{1}:\{0, \ldots, 255\}^{2} \rightarrow[0,1]$, which is defined below, is observed on each of the color components: 
$d_{1}\left(c_{1}, c_{2}\right)=\frac{1}{255}\left|c_{1}-c_{2}\right|$

while on the introduced pixel descriptors, the distance function $d_{2}:[0,1]^{2} \rightarrow[0,1]$ is applied:

$$
d_{2}\left(t_{1}, t_{2}\right)=\left|t_{1}-t_{2}\right|
$$

With intuitively selected values of the parameters $\lambda$ and $\omega$, the distance function $d_{\lambda, \omega}$ is applied in FCM algorithm for the image segmentation. The evaluation of the parameters and quality of the executed segmentation is done by comparing the obtained results with the ones achieved using the Euclidean metric. We use $d_{E}: \bar{P}^{2} \rightarrow[0,1]$ as a referent distance function,

$d_{E}\left(p_{1}, p_{2}\right)=\frac{1}{255 \sqrt{3}} \sqrt{r^{2}+g^{2}+b^{2}}$

where $r=\left|r_{1}-r_{2}\right|, g=\left|g_{1}-g_{2}\right|$ and $b=\left|b_{1}-b_{2}\right|$ , for pixels $p_{i}=\left(r_{i}, g_{i}, b_{i}\right) \in P, i \in\{1,2\}$, and $\bar{P}=\{0, \ldots, 255\}^{3}$.

\section{TEST}

In this test we observe an image that is made by one of the authors with a mobile phone, see Figure 2.

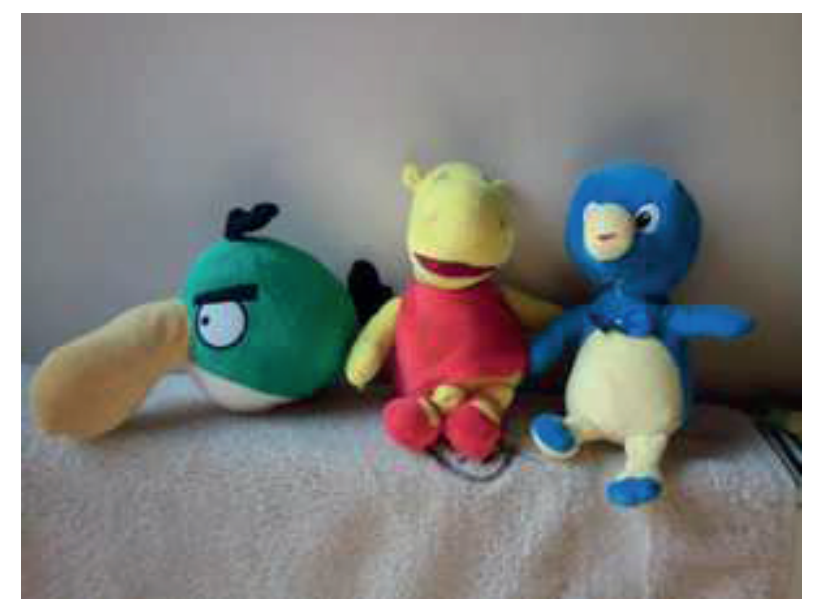

Fig. 2. Original image

The image is $225 \times 300$ pixels in size. The original image is segmented using $d_{E}$ metric and the WAMP aggregated distance function with ten different parameter values, $d_{i}=d_{\Lambda(i) \Omega(i)}$,

$$
\begin{aligned}
& \Lambda(i)=\left\{\lambda_{1}, \lambda_{2}, \lambda_{3}, \lambda_{4}, \lambda_{5}\right\}, \\
& \Omega(i)=\left\{\omega_{1}, \omega_{2}, \omega_{3}, \omega_{4}, \omega_{5}\right\}, \text { see Figure } 3 .
\end{aligned}
$$

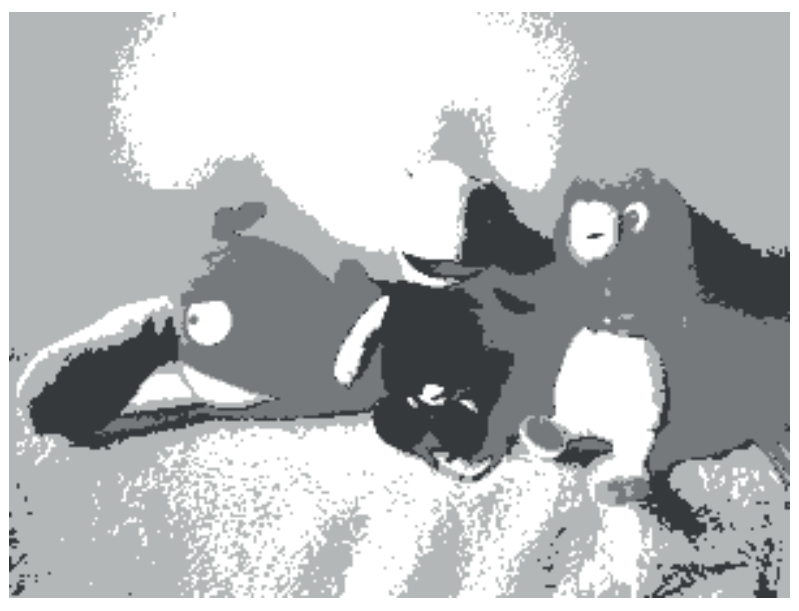

Fig. 3. Segmentation

For many further details see [20].

\section{CONCLUSION}

Based on the applied distance functions and the evaluation parameters obtained in the segmentation we obtained very good results. With suitably selected parameters, WAMP aggregated distance function gives very good results in image segmentation. We can notice that the obtained results are significantly dependent on the values of $\omega$ parameter. Excellent results are produced for $\omega_{i}>1$, while for $\omega_{i}<1$ they are very poor.

Further investigation on applications of extended product operator from Section IV in image segmentation will be managed. It will be examined the possibility of generalizations of $W A M P$ aggregation function with some other types of functions instead of power functions, and their applications in image processing and other fields where decision making based on uncertain information fusion plays a significant role.

\section{ACKNOWLEDGMENT}

Authors acknowledge the financial support of the Ministry of Education, Science and Technological Development of the Republic of Serbia, in the frame of Projects applied under No. 174009 (the first author), 174008 (the second author), TR 34014 (the third author). 


\section{REFERENCES}

[1] M. Grabisch J.-L. Marichal R. Mesiar E. Pap, Aggregation Functions, Cambridge University Press, 2009.

[2] M. Grabisch, J. -L. Marichal, R. Mesiar, E. Pap, "Aggregation functions: Means", Inform. Sci. 181, 2011, pp. 1-22.

[3] M. Grabisch, J. -L. Marichal, R. Mesiar, E. Pap, "Aggregation functions: construction methods, conjunctive, disjunctive and mixed classes", Inform. Sci. 181, 2011, pp. 23-43.

[4] I. J. Rudas, E. Pap, J. Fodor, "Information aggregation in intelligent systems: an application oriented approach", Knowledge Based Systems 38, 2013, pp. 3-13.

[5] I. Bloch, "Fuzzy geodesic distance in images", Fuzzy Logic in Artificial Intelligence Towards Intelligent Systems: IJCAI '95 Workshop Montr' eal, Canada, August 19-21, 1995 Selected Papers, Springer Berlin Heidelberg, 1997, pp. 153-166.

[6] V. Curić, Distance Functions and Their Use in Adaptive Mathematical Morphology, Phd. thesis, Acta Universitatis Upsaliensis 2014.

[7] V. Ćurić, J. Lindblad, N. Sladoje, "Distance measures between digital fuzzy objects and their applicability in image processing", Combinatorial Image Analysis, Springer Berlin Heidelberg, 2011, pp. 385-397.

[8] M. M. Deza, E. Deza, Encyclopedia of Distances, Springer-Verlag Berlin Heidelberg, 2012.

[9] T. Eiter, H. Mannila, "Distance measures for point sets and their computation", Acta Informatica, vol. 34, no. 2, 1997, pp. 109-133.

[10] R. Kimmel, N. Kiryati, A. M. Bruckstein, "Sub-pixel distance maps and weighted distance transforms", J. Mathematical Imaging and Vision, vol. 6, no. 2, 1996, pp. 223-233.

[11] Lj. Nedović, N. M. Ralević, I. Pavkov, "Aggregated distance functions and their application in the fuzzy c-means algorithm", Soft Computing, doi: 10.1007/ s00500-017-2657-9.

[12] Dj. Obradović, Z. Konjović, E. Pap, N. Ralević, "The maximal distance between imprecise point objects", Fuzzy Sets and Syst. 170, 2011, pp. 76-94.
[13] Dj. Obradović, Z. Konjović, E. Pap, I. J. Rudas, "Linear Fuzzy Space Based Road Lane Detection", Knowledge Based Syst. 38, 2013, pp. 37-47.

[14] M.-P. Dubuisson, A. K. Jain, "A modified Hausdorff distance for object matching", Pattern Recognition, 1994. Vol. 1-Conference A: Computer Vision \& Image Processing., Proceedings of the 12th IAPR International Conference on IEEE, vol. 1, 1994, pp. 566-568.

[15] Lj. Nedović, M. Delić, N. M. Ralević, "OWA-aggregated Distance Functions and their Application in Image Segmentation", Proc. of 16th IEEE International Symposium on Intelligent Systems and Informatics (SISY), 2018, pp. 311-316.

[16] Lj. Nedović, M. Delić, "Image segmentation by applying medianaggregated distance functions", Proc. of The Third Conference on Mathematics in Engineering: Theory and Applications, Faculty of technical sciences, University of Novi Sad, 2018, pp. 1-6.

[17] T. Ojala, M. Pietikäinen, D. Harwood, "Performance evaluation of texture measures with classification based on Kullback discrimination of distributions", Proceedings of 12th International Conference on Pattern Recognition, vol. 1, 1994, pp. 582-585.

[18] T. Ojala, M. Pietikäinen, D. Harwood, "A comparative study of texture measures with classification based on featured distributions", Pattern Recognition, Elsevier, vol. 29, no. 1, 1996, pp. 51-59.

[19] M. Delić, J. Lindblad, N. Sladoje, ” LBP - a novel member of the local binary pattern family based on -cutting", 9th International Symposium on Image and Signal Processing and Analysis, ISPA 2015, Zagreb, Croatia, September 7-9, 2015, pp. 13-18.

[20] Lj. Nedović, M. Delić, E. Pap, "Extended powerbased aggregation of distance functions and application in image segmentation", Inform. Sci. https:// doi.org/10.1016/j.ins.2019.04.053.

[21] T. Calvo, G. Mayor, J. Suñer, "Globally Monotone Extended Aggregation Functions", in Enric Trillas: A Passion for Fuzzy Sets: A Collection of Recent Works on Fuzzy Logic, Springer International Publishing, 2015, pp. 49-66.

[22] R. C. Gonzalez, R. E. Woods, Digital Image Processing (3rd Edition), Prentice-Hall, Inc., 2006. 